QUARTERLY OF APPLIED MATHEMATICS

VOLUME LXIV, NUMBER 2

JUNE 2006, PAGES 283-290

$\mathrm{S} 0033-569 \mathrm{X}(06) 01002-5$

Article electronically published on April 6, 2006

\title{
VISCOUS FLOW DUE TO A SHRINKING SHEET
}

\author{
BY \\ M. MIKLAVČIČ (Dept. of Mathematics, Michigan State University, East Lansing, Michigan 48824) \\ AND \\ C. Y. WANG (Dept. of Mathematics, Michigan State University, East Lansing, Michigan 48824)
}

\begin{abstract}
The viscous flow induced by a shrinking sheet is studied. Existence and (non)uniqueness are proved. Exact solutions, both numerical and in closed form, are found.
\end{abstract}

1. Introduction. The viscous flow due to a stretching boundary occurs in expanding or contracting surfaces in a fluid such as extrusion of sheet material from a die and the elongation of pseudopods. Crane $[3$ found a closed form solution for steady, twodimensional stretching where the velocity on the boundary is away and proportional to the distance from a fixed point. The more basic stretching solutions (which differ appreciably from Crane's) are as follows. Gupta and Gupta [4 added suction or injection on the surface. The flow inside a stretching channel or tube was considered by Brady and Acrivos [2] and the flow outside a stretching tube by Wang [10]. The three-dimensional and axisymmetric stretching surface was studied by Wang [9]. The unsteady stretching film was investigated by Wang [11] and Usha and Sridharan [8]. The above solutions are also rare exact similarity solutions of the Navier-Stokes equations.

There is no doubt about the existence of Crane's stretching flow, since an exact closed form solution has been found. The stability was shown by Bhattacharyya and Gupta 11 . The uniqueness of the flow has been proved independently by McLeod and Rajagopal [6] and Troy et al. [7.

On the other hand, very little is known about the shrinking sheet where the velocity on the boundary is towards a fixed point. Only a very specific unsteady shrinking film solution was discussed by Wang [11. From continuity Crane's stretching sheet solution would induce a far field suction towards the sheet, while the shrinking sheet would cause a velocity away from the sheet. Thus from physical grounds vorticity of the shrinking sheet is not confined within a boundary layer, and the flow is unlikely to exist unless adequate suction on the boundary is imposed. The purpose of this paper is to study the

Received July 6, 2005.

2000 Mathematics Subject Classification. Primary 76D03, 76D05, 34B15.

E-mail address: milan@math.msu.edu

E-mail address: cywang@math.msu.edu

(C)2006 Brown University

Reverts to public domain 28 years from publication 
properties of the flow due to a shrinking sheet with suction. Suction occurs when the fluid condenses on the surface, such as in chemical vapor deposition (Jensen et al [5]). We shall prove existence and discuss (non)uniqueness of exact solutions.

2. Formulation. Let $(u, v, w)$ be velocity components in the Cartesian directions $(x, y, z)$ respectively. The continuity and steady constant property Navier-Stokes equations are

$$
\begin{gathered}
u_{x}+v_{y}+w_{z}=0, \\
u u_{x}+v u_{y}+w u_{z}=-p_{x} / \rho+\nu \nabla^{2} u, \\
u v_{x}+v v_{y}+w v_{z}=-p_{y} / \rho+\nu \nabla^{2} v, \\
u w_{x}+v w_{y}+w w_{z}=-p_{z} / \rho+\nu \nabla^{2} w .
\end{gathered}
$$

Here $p$ is the pressure, $\rho$ is the density and $\nu$ is the kinematic viscosity. The boundary conditions on the sheet at $z=0$ are:

$$
u=-a x, \quad v=-a(m-1) y, \quad w=-W
$$

where $a>0$ is the shrinking constant, $W$ is the suction velocity and $m=1$ when the sheet shrinks in the $x$ direction only, $m=2$ when the sheet shrinks axisymmetrically. Far from the sheet the fluid has no lateral velocities and the pressure is uniform. The similarity transformation is

$$
u=a x f^{\prime}(\eta), \quad v=a(m-1) y f^{\prime}(\eta), \quad w=-\sqrt{a \nu} m f(\eta)
$$

where $\eta=z \sqrt{a / \nu}$. Equation (2.1) is automatically satisfied, equation (2.4) becomes

$$
p / \rho=\nu w_{z}-w^{2} / 2+\text { constant }
$$

and equations (2.212.3) reduce to the ordinary differential equation

$$
f^{\prime \prime \prime}+m f f^{\prime \prime}-\left(f^{\prime}\right)^{2}=0 .
$$

The boundary conditions are

$$
f^{\prime}(0)=-1, \quad f(0)=\frac{W}{m \sqrt{a \nu}} \equiv s, \quad f^{\prime}(\infty)=0 .
$$

Equations (2.8,2.9) have never been studied before.

We present here a special exact solution. When

$$
s=\sqrt{\frac{6}{2 m-1}}
$$

equations $(2.8+2.9)$ have an exact solution

$$
f(\eta)=\frac{s^{2}}{\eta+s}
$$

Note that in this case $f(\infty)=0$ and the decay is algebraic. In the rest of the article we will explore the possibility of $f(\infty) \neq 0$. 
3. Characterization of solutions with $f(\infty) \neq 0$. Throughout this section we will assume that for some $\delta \in[-\infty, \infty)$ we have $f \in C^{3}(\delta, \infty)$ such that

$$
f^{\prime \prime \prime}+m f f^{\prime \prime}-\left(f^{\prime}\right)^{2}=0 \quad \text { on } \quad(\delta, \infty)
$$

there exists $x_{0} \in(\delta, \infty)$ such that $f^{\prime}\left(x_{0}\right)<0$ or $f^{\prime \prime}\left(x_{0}\right)>0$,

$$
\lim _{x \rightarrow \infty} f^{\prime}(x)=0,
$$

$$
\text { it is not true that } \lim _{x \rightarrow \infty} f(x)=0 \text {. }
$$

For convenience, we shall assume that $0<m<15$.

Lemma 3.1. There exists $x_{1} \in(-\delta, \infty)$ such that

$$
f(x)>0, f^{\prime}(x)<0, f^{\prime \prime}(x)>0, f^{\prime \prime \prime}(x)<0 \text { for } x>x_{1} .
$$

Proof. Since

$$
\left(f^{\prime \prime} e^{m \int f}\right)^{\prime}=e^{m \int f} f^{\prime 2} \geq 0
$$

we see that $f^{\prime \prime}$ has at most one zero in $(\delta, \infty)$. If there exists $x^{*}$ such that $f^{\prime \prime}\left(x^{*}\right)=0$, then (3.5) implies $f^{\prime \prime}>0$ on $\left(x^{*}, \infty\right)$. If on the other hand $f^{\prime \prime}$ is never 0 , then assumptions (3.2) and (3.3) imply that $f^{\prime \prime}>0$ on $(\delta, \infty)$. In each case there exists $x^{*}$ such that

$$
f^{\prime \prime}(x)>0 \quad \text { for } \quad x>x^{*}
$$

and in view of (3.3) we have that $f^{\prime}(x)<0$ for $x>x^{*}$.

Since

$$
f^{i v}+m f f^{\prime \prime \prime}=(2-m) f^{\prime} f^{\prime \prime}
$$

we have that

$$
\left(f^{\prime \prime \prime} e^{m \int f}\right)^{\prime}=(2-m) f^{\prime} f^{\prime \prime} e^{m \int f} .
$$

Hence, if $m=2$, then $f^{\prime \prime \prime}$ is never 0 and if $m \neq 2$, then $f^{\prime \prime \prime}$ has at most one 0 in $\left(x^{*}, \infty\right)$. (3.3) and (3.6) imply that there exists $x_{1}$ such that $f^{\prime \prime \prime}<0$ on $\left(x_{1}, \infty\right)$. This, (3.1) and (3.6) imply $f>0$ on $\left(x_{1}, \infty\right)$, which completes the proof.

Lemma 3.1 and (3.4) imply

$$
\lim _{x \rightarrow \infty} f(x)=C
$$

for some $C \in(0, \infty)$.

Lemma 3.2. There exists $c_{0} \in(0, \infty)$ such that

$$
f^{\prime \prime}(x)<c_{0} e^{-m C x} \quad \text { for } x>x_{1} .
$$

Proof. Since

$$
f^{\prime \prime \prime}+m C f^{\prime \prime}=f^{\prime 2}-m(f-C) f^{\prime \prime}
$$


Lemma 3.1 implies that for $x_{1}<x<L<\infty$,

$$
\begin{aligned}
f^{\prime \prime}+\left.m C f^{\prime}\right|_{x} ^{L} & =\int_{x}^{L} f^{\prime 2}-m(f-C) f^{\prime \prime} \\
& <\int_{x}^{L} f^{\prime 2} \\
& <-f^{\prime}(x) \int_{x}^{L}-f^{\prime}=-f^{\prime}(x)(f(x)-f(L))
\end{aligned}
$$

and sending $L \rightarrow \infty$ gives

$$
-f^{\prime \prime}(x)-m C f^{\prime}(x)<-f^{\prime}(x)(f(x)-C) .
$$

Since there exists $x^{*}>x_{1}$ such that $0<f(x)-C<m C / 3$ for $x>x^{*}$ we have

$$
-f^{\prime \prime}(x)-\frac{2 m C}{3} f^{\prime}(x)<0
$$

and hence for some $c^{*}<\infty$,

$$
-f^{\prime}(x)<c^{*} e^{-2 m C x / 3} \quad \text { for } x>x^{*} .
$$

Using this in (3.9) gives

$$
f^{\prime \prime}(x) e^{m C x}-f^{\prime \prime}\left(x^{*}\right) e^{m C x^{*}}<\int_{x^{*}}^{x} e^{m C s} f^{\prime}(s)^{2} d s<c^{* 2} \int_{x^{*}}^{\infty} e^{-m C s / 3} d s,
$$

which implies the conclusion of the lemma.

Define a Banach space $X$ to be the set of all $u \in C^{2}[0, \infty)$ such that

$$
\lim _{x \rightarrow \infty} u(x)=\lim _{x \rightarrow \infty} u^{\prime}(x)=0
$$

and

$$
\|u\|=\sup _{x \geq 0}\left|u^{\prime \prime}(x)\right| e^{4 x}<\infty .
$$

For $b \in[0,1]$ and $m \in(0,15)$ define $T_{b, m}: X \rightarrow X$ by

$$
\left(T_{b, m} u\right)^{\prime \prime}(x)=b e^{-4 x}+\int_{0}^{x} e^{4(s-x)}\left(u^{\prime}(s)^{2}-m u(s) u^{\prime \prime}(s)\right) d s .
$$

It is easy to see that if $u \in X$ and $\|u\| \leq 2$, then

$$
\left\|T_{b, m} u\right\| \leq b+(m+1)\|u\|^{2} / 64<2 .
$$

If $u_{1}, u_{2} \in X,\left\|u_{1}\right\| \leq 2$ and $\left\|u_{2}\right\| \leq 2$, then a straightforward calculation gives

$$
\left\|T_{b, m} u_{1}-T_{b, m} u_{2}\right\| \leq \frac{m+1}{16}\left\|u_{1}-u_{2}\right\| .
$$

(3.11) and (3.12) imply

Theorem 3.3. For every $b \in[0,1]$ and $m \in(0,15)$ there exists a unique $U_{b, m} \in X$ such that

$$
\left\|U_{b, m}\right\| \leq 2 \quad \text { and } \quad T_{b, m} U_{b, m}=U_{b, m} .
$$


Note that

$$
U_{b, m}^{\prime \prime \prime}=\left(U_{b, m}^{\prime}\right)^{2}-m\left(U_{b, m}+4 / m\right) U_{b, m}^{\prime \prime}, \quad U_{b, m}^{\prime \prime}(0)=b .
$$

Using the differential equation (3.13) enables us to extend the definition of $U_{b, m}(x)$ for $x<0$ on the maximal interval.

Lemma 3.4. For any $b \in(0,1]$ and $m \in(0,15)$ there exists $\gamma \geq 0$ such that

$$
U_{b, m}(x)=U_{1, m}(x+\gamma) \quad \text { for } x \geq 0 .
$$

Proof. Continuity of $U_{1, m}^{\prime \prime}$ implies that there exists $\gamma \geq 0$ such that $U_{1, m}^{\prime \prime}(\gamma)=b$. Define $v(x)=U_{1, m}(x+\gamma)$ for $x \geq 0$. Since

$$
v^{\prime \prime}(x) e^{4 x}=U_{1, m}^{\prime \prime}(x+\gamma) e^{4(x+\gamma)-4 \gamma} \leq 2
$$

we have that $v \in X$ and $\|v\| \leq 2$. (3.13) implies

$$
v^{\prime \prime \prime}+4 v^{\prime \prime}=v^{2}-m v v^{\prime \prime}, \quad v^{\prime \prime}(0)=b
$$

and integration gives that $T_{b, m} v=v$. Therefore $v=U_{b, m}$ by Theorem 3.3

Lemma 3.5. $U_{1,1}(x)=e^{-4 x} / 16$.

Proof. Let $v(x)=e^{-4 x} / 16$. Note $v \in X$ and $\|v\|=1$. A direct calculation shows $T_{1,1} v=v$; hence, $v=U_{1,1}$ by Theorem 3.3 .

Theorem 3.6. There exist $\beta \in\left(x_{1}, \infty\right)$ and $\gamma \geq 0$ such that

$$
f(x)=C+\frac{m C}{4} U_{1, m}\left(\frac{m C}{4}(x-\beta)+\gamma\right) \quad \text { for } x \geq \beta .
$$

Proof. Choose $\beta>x_{1}$ such that

$$
\left(\frac{4}{m C}\right)^{3} c_{0} e^{-m C \beta} \leq 2 \quad \text { and } \quad\left(\frac{4}{m C}\right)^{3} f^{\prime \prime}(\beta) \leq 1,
$$

where $c_{0}$ is as given in Lemma 3.2. Define

$$
v(x)=\frac{4}{m C} f\left(\frac{4}{m C} x+\beta\right)-\frac{4}{m} \quad \text { for } x \geq 0 .
$$

Note

$$
v^{\prime \prime}(x) e^{4 x}=\left(\frac{4}{m C}\right)^{3} f^{\prime \prime}\left(\frac{4}{m C} x+\beta\right) e^{m C(4 x /(m C)+\beta)-m C \beta} \leq\left(\frac{4}{m C}\right)^{3} c_{0} e^{-m C \beta} \leq 2 .
$$

Hence $v \in X$ and $\|v\| \leq 2$.

Since $v$ satisfies (3.14), an integration gives that $T_{b, m} v=v$, where

$$
b=v^{\prime \prime}(0)=\left(\frac{4}{m C}\right)^{3} f^{\prime \prime}(\beta) \leq 1
$$

and therefore Theorem 3.3 implies that $v=U_{b, m}$. Lemma 3.4 implies that

$$
v(x)=U_{1, m}(x+\gamma),
$$

and this completes the proof. 
4. Results and conclusions. Theorem 3.6 implies that every solution of our boundary value problem (2.8) and (2.9), for which $f(\infty) \neq 0$, is of the form

$$
f(x)=C+\frac{m C}{4} U_{1, m}\left(\frac{m C}{4} x+\gamma\right) \quad \text { for } x \geq 0
$$

for some constants $C>0$ and $\gamma . U_{1, m}$ is given by Theorem 3.3 and extended to the maximal interval by (3.13). The boundary condition $f(0)=s$ implies

$$
C+\frac{m C}{4} U_{1, m}(\gamma)=s
$$

and $f^{\prime}(0)=-1$ implies

$$
\left(\frac{m C}{4}\right)^{2} U_{1, m}^{\prime}(\gamma)=-1 .
$$

Equations (4.1, 4.2) imply that

$$
s=\frac{4+m U_{1, m}(\gamma)}{m \sqrt{-U_{1, m}^{\prime}(\gamma)}} .
$$

Plotting the right-hand side of (4.3) vs. $\gamma$ gives all possible $s$ and their multiplicity.

When $m=1$, Lemma 3.5 implies $U_{1,1}(x)=e^{-4 x} / 16$; hence (4.3) becomes

$$
s=2 \cosh (2 \gamma+\ln 8) \text {. }
$$

Therefore, there are no solutions for $s<2$, one for $s=2$ and two for $s>2$. There is an additional, algebraically decaying solution (2.11) when $s=\sqrt{6}$. Equations (4.2, 4.3) imply that $s=C+1 / C$. This is summarized in Figure 1.

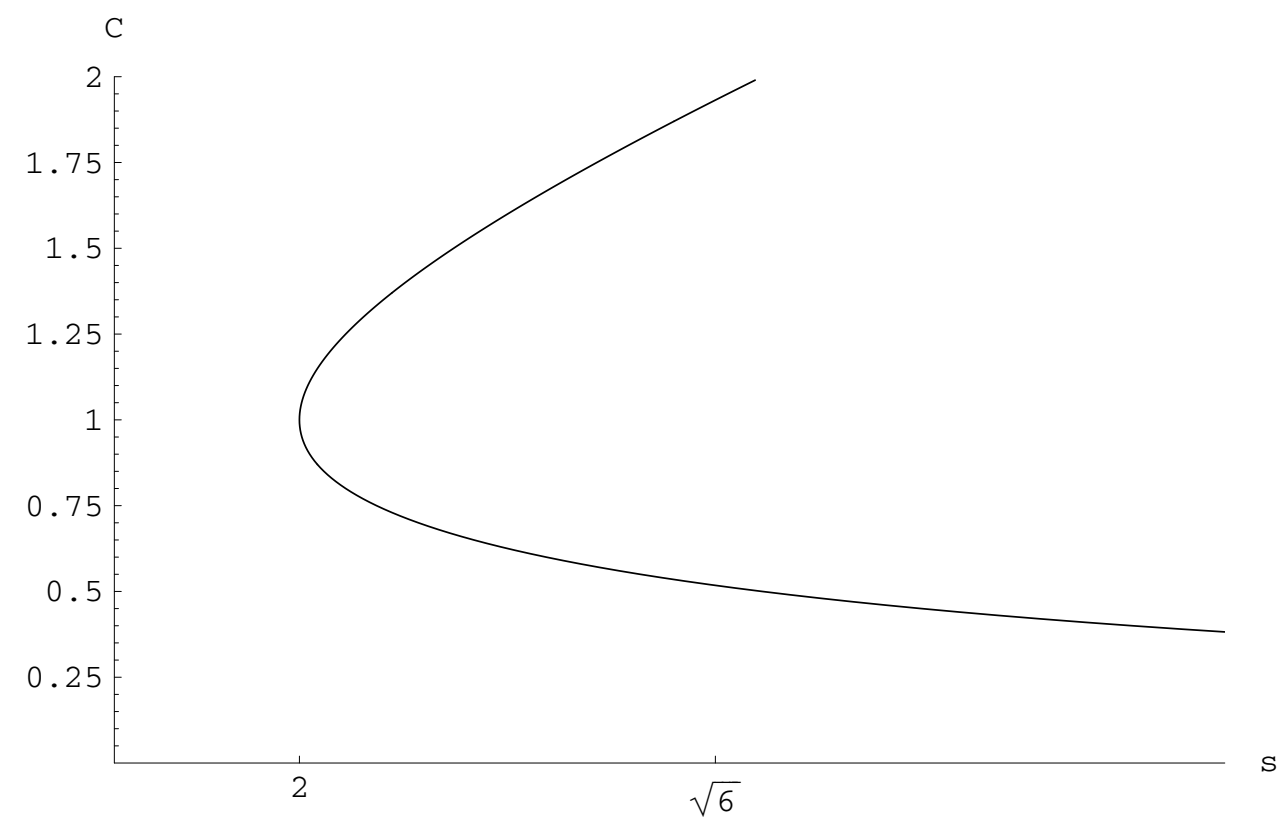

Fig. 1. $C$ vs. $s$ when $m=1$ 
When $m=2, U_{1,2}$ is known only numerically. One can find it also by solving (3.13) as an initial value problem with

$$
U_{1,2}(0)=0.061780044595, \quad U_{1,2}^{\prime}(0)=-0.2480768280953, \quad U_{1,2}^{\prime \prime}(0)=1 .
$$

At first glance it behaves rather uninterestingly: monotone decreasing, it blows up at $\delta_{0} \doteq-1.303249602$. However, a parametric plot of $(s(\gamma), C(\gamma))$ for $\gamma>\delta_{0}$ reveals an interesting graph; see Figure 2. Note first that for $s<s_{0} \doteq 1.31175869$ we have

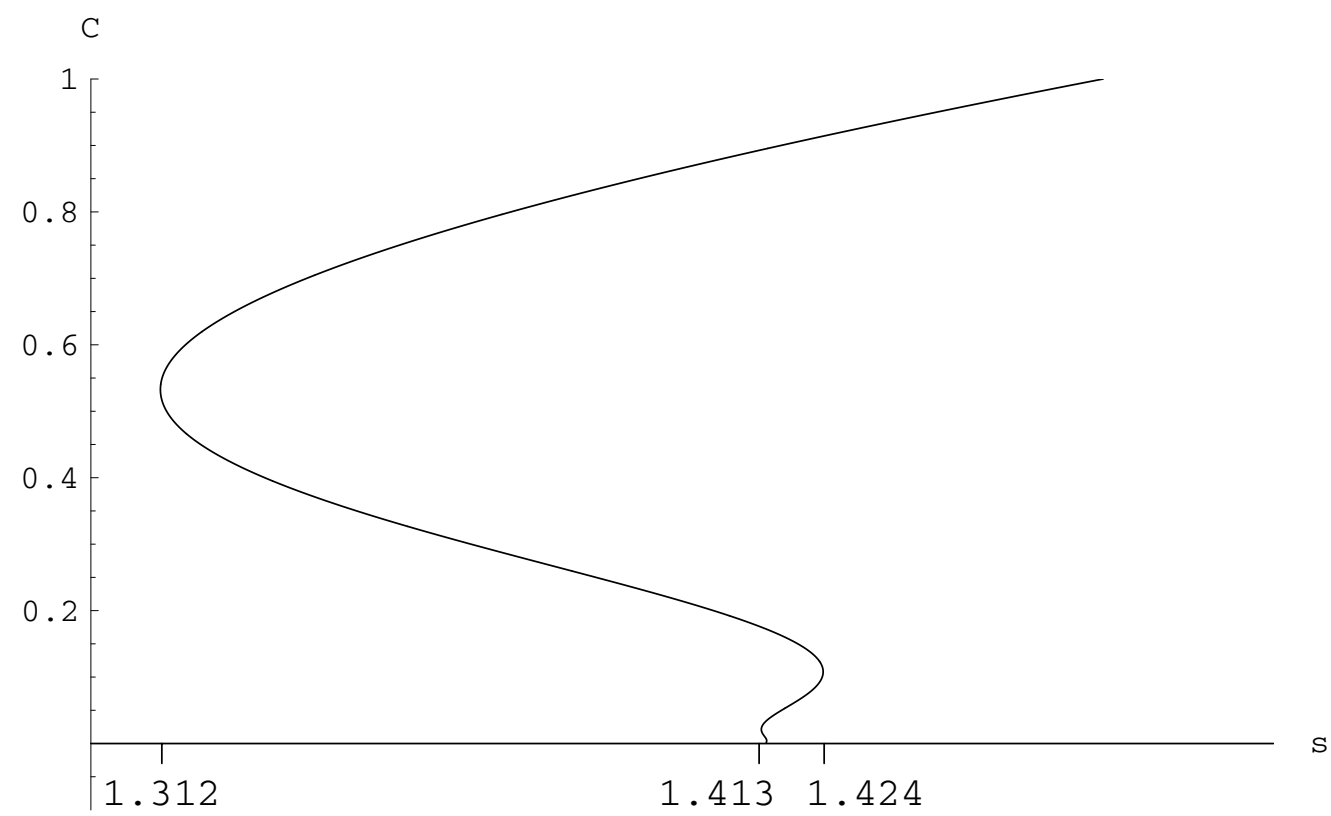

FIG. 2. $C$ vs. $s$ when $m=2$. As $C \rightarrow 0$ the curve converges to $s=\sqrt{2}$; however, it does cross the limiting value infinitely many times.

no solution. For $s>s_{1} \doteq 1.42382978$ we have only one solution. For $s_{0}<s<s_{2} \doteq$ 1.41337508 we have two solutions. However, for $s_{2}<s<s_{1}$ things are quite complicated. It turns out that the behaviour of $s$ as $C \rightarrow 0$ is governed by details of how $U_{1,2}(\gamma)$ blows up as $\gamma \rightarrow \delta_{0}$. Numerically, we first found out that

$$
U_{1,2}(\gamma)-\frac{2}{\gamma-\delta_{0}}+2 \rightarrow 0 \quad \text { as } \gamma \rightarrow \delta_{0} .
$$

If we now let $\phi(\gamma)=U_{1,2}(\gamma)+2-2 /\left(\gamma-\delta_{0}\right)$ and use (3.13), neglecting quadratic terms in $\phi$, we obtain a much better approximation of $U_{1,2}(\gamma)$ for $\gamma$ near $\delta_{0}$ :

$$
U_{1,2}(\gamma) \doteq \frac{2}{\gamma-\delta_{0}}-2+\sqrt{\gamma-\delta_{0}}\left(a_{1} \cos \left(a_{0} \ln \left(\gamma-\delta_{0}\right)\right)+a_{2} \sin \left(a_{0} \ln \left(\gamma-\delta_{0}\right)\right)\right),
$$

where $a_{0}=\sqrt{15} / 2, a_{1}=0.13946$ and $a_{2}=0.49590$. Using this approximation in (4.3) gives for small $\gamma-\delta_{0}$ that

$$
s \doteq \sqrt{2}\left(1+\left(\gamma-\delta_{0}\right)^{3 / 2}\left(a_{1}^{*} \cos \left(a_{0} \ln \left(\gamma-\delta_{0}\right)\right)+a_{2}^{*} \sin \left(a_{0} \ln \left(\gamma-\delta_{0}\right)\right)\right)\right)
$$


where $a_{1}^{*}=0.397136$ and $a_{2}^{*}=0.4903$. Hence if $s_{n}$ denote the extreme values of $s$, then we have $n$ solutions whenever

$$
s_{n-2}<s<s_{n} \text { and } n \geq 2 \text { is even }
$$

or

$$
s_{n}<s<s_{n-2} \text { and } n \geq 3 \text { is odd. }
$$

Using (4.8) one can show that

$$
s_{n} \doteq \sqrt{2}\left(1-(-1)^{n} e^{-2.563 \ldots-3 n \pi / \sqrt{15}}\right) \quad \text { for } n \text { large, }
$$

which is actually a very good approximation of $s_{n}$ for $n$ as low as 3 . Note that, when $s=\sqrt{2}$, the algebraically decaying solution (2.11) with $C=0$ is also possible.

We find for both two-dimensional and axisymmetric shrinking sheets that there must be adequate suction on the surface to maintain the flow. At certain suction rates the solution may not be unique. Which solution actually occurs depends on the flow stability, which is not investigated in this paper. There are also significant differences as can be seen from a comparison of Figures 2 and 1. As $C \rightarrow 0, s$ becomes infinite for the twodimensional case but $s$ remains finite for the axisymmetric case. On the other hand, for the axisymmetric case there exist infinitely many solutions near $s=\sqrt{2}$. Since in that neighborhood solutions are quite sensitive to initial conditions, a certain degree of chaos may occur.

For the stretching sheet, studied previously, solutions are unique and exist for all suction (injection) rates. The shrinking sheet studied in this paper offers a wealth of nonlinear fluid phenomena.

\section{REFERENCES}

[1] Bhattacharyya, S.N. and Gupta, A.S. (1985) On the stability of viscous flow over a stretching sheet. Quart. Appl. Math. 43, 359-367. MR0814233 (87a:76056)

[2] Brady, J.F. and Acrivos, A. (1981) Steady flow in a channel or tube with accelerating surface velocity. An exact solution to the Navier-Stokes equations with reverse flow. J. Fluid Mech. 112, 127-150. MR0639234 (82m:76017)

[3] Crane, L.J. (1970) Flow past a stretching plate. ZAMP 21, 645-647.

[4] Gupta, P.S. and Gupta, A.S. (1977) Heat and mass transfer on a stretching sheet with suction and blowing. Can. J. Chem. Eng. 55, 744-746.

[5] Jensen, K.F., Einset, E.O. and Fotiadis, D.I. (1991) Flow phenomena in chemical vapor deposition of thin films. Ann. Rev. Fluid Mech. 23, 197-232.

[6] McLeod, J.B. and Rajagopal, K.R. (1987) On the uniqueness of flow of a Navier-Stokes fluid due to a stretching boundary. Arch. Rat. Mech. Anal. 98, 385-393. MR0872753 (88c:35131)

[7] Troy, W., Overman II, E.A., Ermentrout, G.B. and Keener, J.P. (1987) Uniqueness of flow of a second-order fluid past a stretching sheet. Quart. Appl. Math. 44, 753-755. MR0872826|(87m:76009)

[8] Usha, R. and Sridharan, R. (1995) The axisymmetrical motion of a liquid film on an unsteady stretching surface. J. Fluids Eng. 117, 81-85.

[9] Wang, C.Y. (1984) The three-dimensional flow due to a stretching flat surface. Phys. Fluids 27, 1915-1917. MR0758728 (85g:76014)

[10] Wang, C.Y. (1988) Fluid flow due to a stretching cylinder. Phys. Fluids 31, 466-468.

[11] Wang, C.Y. (1990) Liquid film on an unsteady stretching sheet. Quart. Appl. Math. 48, 601-610. MR:1079908 (91h:76025) 